\title{
On the solutions of the Cartan equation in Metric Affine Gravity
}

\author{
R. Mignani*and R.Scipioni ${ }^{\circ}$ \\ ${ }^{(*)}$ Dipartimento di Fisica "E. Amaldi" \\ Universita' di Roma "Roma Tre" \\ Via della Vasca Navale, 84 \\ 00146 Roma, Italy* \\ (०)Department of Physics and Astronomy \\ The University of British Columbia \\ 6224 Agricultural Road \\ Vancouver, B.C., Canada V6T 1Z1 ${ }^{\dagger}$
}

October 29, 2018

\begin{abstract}
In the Tucker-Wang approach to Metric Affine gravity we review some particular solutions of the Cartan equation for the non-riemannian part of the connection. As application we show how a quite general non Riemannian model gives a Proca type equation for the trace of the nonmetricity 1-forms $Q$.
\end{abstract}

*mignani@fis.uniroma3.it

${ }^{\dagger}$ scipioni@physics.ubc.ca 


\section{INTRODUCTION}

Einstein's theory of gravity which was developed more than eighty years ago provides an elegant and powerful formulation of gravitation in terms of a pseudo-Riemannian geometry. In the variational approach, Einstein's equations are obtained by considering variations, with respect to the metric, of the Hilbert-Einstein action, i.e. the integral of the curvature scalar, associated with the Levi-Civita connection, on the spacetime-volume form . Einstein assumed that the connection was metric compatible and torsion free; a position which is natural but not always convenient. In particular, a number of recent developments in physics suggest the possibility that the treatment of spacetime might involve more than just a Riemannian structure. Let us quote some of them:

1] The vain effort to quantise gravity, which is perhaps so far the strongest piece of evidence for going beyond a geometry dominated by the classical concept of distance.

2] The generalisation of the theory of elastic continua with structure to 4-dimensional spacetime, that provides physical interpretations of the nonRiemannian structures which emerge in the theory $[1,2]$.

3] The description of hadronic (or nuclear) matter in terms of extended structures $[3,4]$.

4] The study of the early universe, in particular singularity theorems, the problem of the unification of interactions and the related problem of compactification of dimensions, and models of inflation with dilaton-induced Weyl covector [5].

Moreover, at the level of the so called string theories there are hints [6-9] that by using non-Riemannian geometry we may accommodate the several degrees of freedom coming from the low-energy limit of string interactions in terms of a non metric-compatible connection with torsion. It is interesting to observe that, since string theories are expected to produce effects which are at least in principle testable at low energies, there may be chances to obtain non-Riemannian models with predictions which can somehow be tested; 
some models may have even some effects on astronomical scales [10-20]. For instance, recently models have been proposed that permit to account for the so-called dark matter by invoking non-Riemannian gravitational interactions [21]. There are several approaches to non-Riemannian gravity: perhaps one of the most popular is that which uses gauge field theories [22-25].

Soon after Einstein proposed his gravitational theory, Weyl found an extension to it, able to include electromagnetism in a unified way [26]. Weyl's theoretical concept was the so called gauge invariance of length. To that purpose, Weyl extended the geometry of spacetime from the Levi-Civita connection to a new space ("Weyl space") with an additional covector $Q=Q_{a} e^{a}$ , where $e^{a}$ denotes the field of coframes of the four-dimensional manifold.

The Weyl connection 1-form reads:

$$
\Gamma_{\alpha \beta}^{W}=\Gamma_{\alpha \beta}+\frac{1}{2}\left(g_{\alpha \beta} Q-e_{\alpha} Q_{\beta}+e_{\beta} Q_{\alpha}\right)
$$

The Weyl form is related to the so-called non-metricity of the spacetime. If we write the interval in the form:

$$
d s^{2}=g_{\mu \nu} d x^{\mu} d x^{\nu}
$$

the square length of a generic vector $V$ can be written as:

$$
V^{2}=g_{\mu \nu} V^{\mu} V^{\nu}=g(V, V)
$$

where $g$ is the $(2,0)$ symmetric tensor defined by:

$$
g=g_{\mu \nu} e^{\mu} \otimes e^{\nu}
$$

We find that the covariant derivative of $V^{2}$ with respect to a generic vector $X$ gives:

$$
\nabla_{X} V^{2}=\left(\nabla_{X} g\right)(V, V)=Q_{a b}(X) V^{a} V^{b}
$$

where $Q_{a b}(X)=\left(\nabla_{X} g\right)\left(X_{a}, X_{b}\right), Q^{a}{ }_{a}=Q$ and we assumed that $\nabla_{X} V=0$. In a spacetime with nonmetricity, the length of a vector changes if we parallely transport the vector along a curve whose tangent vector is $X$.

In Weyl's theory the field $Q$ is identified with the electromagnetic potential A.

Subsequently it was found that Weyl's theory is not viable. However the concept of gauge invariance survived. In particular the concept of local gauge 
invariance flourished in the field of theoretical particle physics. Consider a particle, described, in quantum mechanics, by a wave function $\Psi$. Then, as is well known, postulating the local invariance of the theory under the $U(1)$ abelian group, namely

$$
\Psi \rightarrow e^{i \alpha(x)} \Psi
$$

(with $\alpha(x)$ a function of spacetime coordinates), permits to get the electromagnetic interaction, and therefore to construct the whole classical DiracMaxwell theory for a charged particle in an electromagnetic field.

In 1954 Yang and Mills generalised the abelian $U(1)$ gauge invariance to non-Abelian $S U(2)$-gauge invariance using the approximate conservation of the isotopic spin current as starting point.

In any case it is interesting to observe that the gauge principle originated from General Relativity.

Nowadays the notion of gauge symmetry is one of the cornerstones of theoretical physics; the three non-gravitational interactions are described by means of gauge theories in the framework of the Standard Model.

Thanks to the works of Utiyama, Sciama and Kibble $[25,27,28]$ it was realized that also gravitation can be formulated as a gauge theory, the gauge group being in that case the Poincare" group, that is the semidirect product of the translation and the Lorentz group.

More recently a quite general gauge theory has been formulated which includes General Relativity as a particular case; in this case the gauge group is the so called affine group resulting from the semidirect product of the translation and the general linear group $G L(n, R)$. This theory is called Metric Affine Gravity [22], and it allows for the introduction of a general non-Riemannian connection.

Though it is possible to treat gravity using the gauge approach, it is necessary to remember that in the case of gravitation, contrary to the case of strong and electroweak interactions, we are considering an external symmetry group, i.e. a group acting on spacetime. So a procedure is necessary to mediate the transition from the internal structure, which is proper of any gauge formulation, and the external structures, and to project geometric gauge structures on the base manifold in order to induce gravity. It is presently unclear how this procedure applied to any affine frame takes place. This issue is somewhat similar to the compactification of higher-dimensional supergravity or string theories. 
Recently a different approach to metric affine gravity has been proposed by Tucker and Wang [29-31] based on the metric $\mathbf{g}$ and the connection $\nabla$ as independent variables. Instead of working with the group $G L(4, \mathbf{R})$ (the general linear group), it relies on the definition of torsion and non-metricity in terms of $\mathbf{g}$ and $\nabla$.

The study of non-Riemannian theories of gravitation is in general quite complicate and a powerful formalism is needed in order to simplify calculations. To this purpose, the frame-independent approach to differential geometry seems quite appropriate. This approach has several advantages. Indeed, we do not need to consider particular reference systems or co-frames and the results are unambiguous and easy to apply, bearing in mind that whenever requested, transition to the more traditional component manipulation is possible ( taking into account the fact that since the theory is non-metric, raising and lowering of indices must be done with care).

Aim of this paper is to give an introduction to Metric Affine Gravity in the Tucker-Wang approach. In particular, we shall review some particular solutions of the Cartan equation for the non-riemannian part of the connection. An application will be also given by deriving a Proca-type equation for the trace of the non-metricity 1-forms. We shall consider only the Cartan sector of the theory. This equivalence has been proved for the Einstein sector too [34].

The content of the paper is as follows. Some basic concepts and tools of non riemannian geometry in the frame-independent formalism are reviewed in sect. 2 . In sect. 3 we describe the variational techniques using a tensorial approach. In sect. 4 some particular solutions of the Cartan equation are obtained and classified. This classification will be necessary to prove (in sect. 5 ) that a quite general model of non-Riemannian gravity yelds a Proca- type equation for the Weyl 1-form $Q$. 


\section{NON-RIEMANNIAN GEOMETRY}

In this section a brief introduction is given of the non-Riemannian geometry in the frame independent approach.

The formalism we will be using takes into account the fact that in general we will consider non-metric theories, i.e. theories in which the metric is not regarded any longer as covariantly constant. It is clear that we need to use a formalism which makes use of as less number of indices as possible. This is obtained by formulating the non-Riemannian geometry in the frame independent approach.

One of the fundamental concepts in differential geometry is of Parallel Transport. To define parallel transport we need to introduce a linear connection, a type preserving derivation on the algebra of tensors fields commuting with contractions. We will denote such a connection by $\nabla$. We can specify the most general linear connection by calculating its effects on an arbitrary local basis of vector fields $X_{a}$

$$
\nabla_{X_{a}} X_{b}=\Lambda_{b}^{c}\left(X_{a}\right) X_{c}
$$

where $\Lambda_{b}^{a}$ are a set of $n^{2} 1$-forms, and $n$ is the dimension of the manifold. It is possible to specify a general connection by giving a $(2,0)$ metric symmetric tensor $\mathbf{g}$, a $(2,1)$ tensor $\mathbf{T}$ defined by

$$
\mathbf{T}(X, Y)=\nabla_{X} Y-\nabla_{Y} X-[X, Y]
$$

(with $X, Y$ vector fields) and a $(3,0)$ tensor $\mathbf{S}$ symmetric in the last two arguments. $\mathbf{T}$ is the torsion associated with $\nabla$ and $\mathbf{S}$ is taken to be the metric gradient, $\mathbf{S}=\nabla \mathbf{g}$. Then it is possible to calculate the connection as a function of $\mathbf{g}, \mathbf{S}, \mathbf{T}$. Indeed, by using the relation

$$
X(\mathbf{g}(Y, Z))=\mathbf{S}(X, Y, Z)+\mathbf{g}\left(\nabla_{X} Y, Z\right)+\mathbf{g}\left(Y, \nabla_{X} Z\right)
$$

we obtain

$$
\begin{aligned}
2 \mathbf{g}\left(Z, \nabla_{X} Y\right)= & X(\mathbf{g}(Y, Z))+Y(\mathbf{g}(Z, X))-Z(\mathbf{g}(X, Y))-\mathbf{g}(X,[Y, Z]) \\
& -\mathbf{g}(Y,[X, Z])-\mathbf{g}(Z,[Y, X])-\mathbf{g}(X, \mathbf{T}(Y, Z))-\mathbf{g}(Y, \mathbf{T}(X, Z))- \\
& \mathbf{g}(Z, \mathbf{T}(Y, X))-\mathbf{S}(X, Y, Z)-\mathbf{S}(Y, Z, X)+\mathbf{S}(Z, X, Y)
\end{aligned}
$$


where $X, Y, Z$ are any vector fields.

We define the general curvature operator as:

$$
\mathbf{R}_{X, Y} Z=\nabla_{X} \nabla_{Y} Z-\nabla_{Y} \nabla_{X} Z-\nabla_{[X, Y]} Z
$$

which is a type preserving tensor derivation on the algebra of tensor fields. The $(3,1)$ curvature tensor is defined by:

$$
\mathbf{R}(X, Y, Z, \beta)=\beta\left(R_{X, Y} Z\right)
$$

with $\beta$ an arbitrary 1-form. We can introduce the following set of local curvature 2 -forms $R_{b}^{a}$ :

$$
R_{b}^{a}(X, Y)=\frac{1}{2} \mathbf{R}\left(X, Y, X_{b}, e^{a}\right)
$$

where $e^{a}$ is any local basis of 1 -forms dual to $X_{c}$. We have $e^{a}\left(X_{b}\right)=\delta^{a}{ }_{b}$ or by using the contraction operator with respect to $X, i_{X_{b}}\left(e^{a}\right)=e^{a}\left(X_{b}\right)=\delta^{a}{ }_{b}$. In terms of the connections forms we can write:

$$
R_{b}^{a}=d \Lambda_{b}^{a}+\Lambda_{c}^{a} \wedge \Lambda_{b}^{c}
$$

In a similar manner, the torsion tensor gives rise to a set of local 2-forms $T^{a}$

$$
T^{a}(X, Y) \equiv \frac{1}{2}\left(e^{a}(T(X, Y))\right.
$$

which can be written:

$$
T^{a}=d e^{a}+\Lambda_{b}^{a} \wedge e^{b}
$$

By using the symmetry of the tensor $\mathbf{g}$, the tensor $\mathbf{S}$ can be used to define the local non-metricity 1-forms $Q_{a b}$ symmetric in their indices:

$$
Q_{a b}(Z)=\mathbf{S}\left(Z, X_{a}, X_{b}\right)
$$

It is convenient very often to make use of the exterior covariant derivative $D$.

With $g_{a b} \equiv \mathbf{g}\left(X_{a}, X_{b}\right)$ we get that:

$$
\begin{aligned}
Q_{a b} & =D g_{a b} \\
Q^{a b} & =-D g^{a b}
\end{aligned}
$$


As usual, indices are raised and lowered by means of the components of the metric in a certain local basis. We denote the metric trace of these forms as:

$$
Q=Q_{a}^{a}
$$

We call $Q$ the Weyl 1-form.

In Riemannian geometry we require that the connection be metric compatible $\left(Q_{a b}=0\right.$, or equivalently $\left.\mathbf{S}=0\right)$ and $\mathbf{T}=0$.

It is possible to decompose the connection $\nabla$ into parts that depend on the Levi-Civita connection $\stackrel{\mathrm{o}}{\nabla}$. To this aim we introduce the tensor $\lambda$

$$
\lambda(X, Y, \beta)=\beta\left(\nabla_{X} Y\right)-\beta\left(\stackrel{\mathbf{o}}{\nabla}_{X} Y\right)
$$

for arbitrary vector fields $X, Y$ and 1 -form $\beta$.

To the decomposition above there corresponds a splitting of the connection 1-form into its Riemannian and non-Riemannian parts $\Omega^{a}{ }_{b}$ and $\lambda_{b}^{a}$, respectively, as:

$$
\Lambda_{b}^{a}=\Omega_{b}^{a}+\lambda_{b}^{a}
$$

where

$$
\lambda_{b}^{a} \equiv \lambda\left(-, X_{b}, e^{a}\right)
$$

In terms of these forms we find:

$$
\begin{aligned}
T^{a} & =\lambda^{a}{ }_{c} \wedge e^{c} \\
Q_{a b} & =-\left(\lambda_{a b}+\lambda_{b a}\right)
\end{aligned}
$$

Using relations (11) and (17) we get $Q_{a b}=\mathbf{S}\left(-, X_{a}, X_{b}\right)=-\left(\lambda_{a b}+\lambda_{b a}\right)$, and therefore:

$$
\mathbf{S}=0 \leftrightarrow \lambda_{a b}=-\lambda_{b a}
$$

namely the metric compatibility requires the antisymmetry of $\lambda$.

We define the 1-form $T$ by:

$$
T=i_{a} T^{a}
$$

with $i_{a} \equiv i_{X_{a}}$. We have the following relation:

$$
e^{c} \wedge \star T_{c}=-\star T
$$

where use has been made of the property $\star\left(A \wedge e^{a}\right)=i^{a} \star A$, with $A$ a generic form and $\star$ denotes the Hodge operation associated with the metric $\mathbf{g}$. 
Since a general connection is neither symmetric nor antisymmetric, particular care has to be taken when writing the indices, because in general $\lambda^{a}{ }_{b}$ is different from $\lambda_{b}{ }^{a}$.

Observe that a Riemannian connection $\Omega^{a}{ }_{b}$ being torsion free implies:

$$
d e^{a}+\Omega_{b}^{a} \wedge e^{b}=0
$$

It follows from relation (4) that:

$$
2 \Omega_{a b}=\left(g_{a c} i_{b}-g_{b c} i_{a}+e_{c} i_{a} i_{b}\right) d e^{c}+\left(i_{b} d g_{a c}-i_{a} d g_{b c}\right) e^{c}+d g_{a b}
$$

and

$$
2 \lambda_{a b}=i_{a} T_{b}-i_{b} T_{a}-\left(i_{a} i_{b} T_{c}+i_{b} Q_{a c}-i_{a} Q_{b c}\right) e^{c}-Q_{a b}
$$

The field equations of the Einstein theory are obtained as variational equations deduced from the Einstein-Hilbert action, this being the integral of the curvature scalar of the Levi-Civita connection with respect to the volume form.

The scalar curvature is obtained by contracting the Ricci-tensor, which is the trace of the curvature tensor.

In general we can define two types of tensors:

$$
\boldsymbol{R i c}(X, Y)=e^{a}\left(\mathbf{R}_{X_{a} X} Y\right)
$$

and

$$
\operatorname{ric}(X, Y)=e^{a}\left(\mathbf{R}_{X Y} X_{a}\right)
$$

We have:

$$
\begin{aligned}
\operatorname{Ric}_{c b} & =R_{a c b}{ }^{a} \\
\operatorname{ric}_{c b} & =R_{c b a}{ }^{a}
\end{aligned}
$$

where:

$$
R_{a c b}^{d}=\mathbf{R}\left(X_{a}, X_{c}, X_{b}, e^{d}\right)
$$

The first one has no symmetry in general while ric is a 2 -form which can be shown to be:

$$
\text { ric }=2 R^{a}{ }_{a}=-d Q
$$

Indeed from relations (15), (22), (23) we get:

$$
2 \Lambda=2 \Lambda^{a}{ }_{a}=-Q
$$


whereas relations $(6,7)$ and (25) imply

$$
\operatorname{ric}=2 R_{a}^{a}=2 d \Lambda=-d Q
$$

since $\Lambda_{c}^{a} \wedge \Lambda_{a}^{c}=-\Lambda_{a}^{c} \wedge \Lambda_{c}^{a}=0$.

In the Riemannian case $\mathbf{r i c}=0$, and $\operatorname{Ric}(X, Y)$ goes into the usual Ricci tensor which is symmetric in the two arguments (by virtue of the fact that non-metricity is zero); moreover the Riemann curvature tensor satisfies the antisymmetry property

$$
R_{a b c}{ }^{d}=-R_{a b d}^{c}
$$

The symmetric part of Ric can be contracted with the metric tensor to obtain a generalised curvature:

$$
R=\operatorname{Ric}\left(X_{a}, X_{b}\right) \mathbf{g}\left(X^{a}, X^{b}\right)=\mathbf{R i} c\left(X_{a}, X^{a}\right)
$$

It is possible to obtain the expression for the general $\operatorname{Ric}(X, Y)$ as:

$$
\operatorname{Ric}\left(X_{a}, X_{b}\right)=\stackrel{o}{\operatorname{Ric}}\left(X_{a}, X_{b}\right)+i_{a} i_{c}\left(\stackrel{o}{D} \lambda^{c}{ }_{b}+\lambda^{c}{ }_{d} \wedge \lambda_{b}^{d}\right)
$$

where $\stackrel{o}{D}$ is the covariant exterior derivative with respect to the Levi-Civita connection $\Omega_{b}^{a}$ and

$$
R=\stackrel{o}{R}+i_{a} i_{c}\left(\stackrel{o}{D} \lambda^{c a}+\lambda^{c}{ }_{d} \wedge \lambda^{d a}\right)
$$

If we define the Ricci 1-forms by

$$
P_{b}=i_{a} R_{b}^{a}
$$

one has

$$
P_{a}=\operatorname{Ric}\left(X_{b}, X_{a}\right) e^{b}
$$

and

$$
R=i^{b} P_{b}=i^{b} i_{a} R_{b}^{a}
$$

so that the curvature scalar can be written in general as:

$$
R=2 R^{d}{ }_{c} \otimes e^{c} \otimes X_{d}
$$




\section{Variations of the Generalised Einstein-Hilbert Action}

A non-Riemannian geometry is specified when we give a metric $\mathbf{g}$ and a connection $\nabla$.

In a local coframe $e^{a}$ with dual frame $X_{b}$ such that $e^{a}\left(X_{b}\right)=\delta^{a}{ }_{b}$, the connection forms satisfy $\left(\Lambda_{b}^{a} \equiv \omega^{a}{ }_{b}\right)$ :

$$
\omega^{c}{ }_{b}\left(X_{a}\right) \equiv e^{c}\left(\nabla_{X_{a}} X_{b}\right)
$$

In the following we use orthonormal frames so that:

$$
\mathbf{g}=\eta_{a b} e^{a} \otimes e^{b}
$$

with $\eta_{a b}=\operatorname{diag}(-1,1,1,1, \ldots)$.

It is important to observe that the position (40) permits to transfer the functional dependence on the metric $\mathbf{g}$ to the coframe $e^{a}$. Since in general in the metric affine gauge theory of gravity [22] the metric $g_{a b}$ is considered as a gauge potential independent on $e^{a}$, the assumption that $g_{a b}=\eta_{a b}$ is equivalent to choose a certain gauge as well as requiring that $\mathbf{g}$ depends only on $e^{a}$. We will call it the Tucker-Wang gauge [30,31].

Let us consider an action written in the form:

$$
S[\mathbf{e}, \omega]=\int \Lambda(\mathbf{e}, \omega)
$$

for some $n$-form $\Lambda$.

The field equations of the theory follow from $(\bmod d)$ :

$$
\begin{aligned}
& \underbrace{\Lambda}_{\text {e }}=0 \\
& \underbrace{\Lambda}_{\omega}=0
\end{aligned}
$$

The general curvature scalar is:

$$
R=i^{b} i_{a} R_{b}^{a}
$$

The generalised Einstein-Hilbert action density $\Lambda_{E H}=R \star 1$ can be written

$$
\Lambda_{E H} \equiv R \star 1=\left(i^{b} i_{a} R_{b}^{a}\right) \star 1=R_{b}^{a} \wedge \star\left(e_{a} \wedge e^{b}\right)
$$


¿From the definition of curvature 2-forms it follows that:

$$
\underbrace{R \star 1}_{\omega}=(\underbrace{d \omega_{b}^{a}+\omega^{a}{ }_{c} \wedge \omega_{b}^{c}}_{\omega}) \wedge \star\left(e_{a} \wedge e^{b}\right)
$$

We can write:

$$
d\left(\omega^{a}{ }_{b} \wedge \star\left(e_{a} \wedge e^{b}\right)\right)=d \omega^{a}{ }_{b} \wedge \star\left(e_{a} \wedge e^{b}\right)-\omega^{a}{ }_{b} \wedge d\left(\star\left(e_{a} \wedge e^{b}\right)\right)
$$

so that:

$$
\begin{aligned}
\underbrace{\Lambda_{E H}}_{\omega}= & \dot{\omega}^{a}{ }_{b} \wedge d \star\left(e_{a} \wedge e^{b}\right)+(\underbrace{\omega_{c}^{a} \wedge \omega_{b}^{c}}_{\omega}) \wedge \star\left(e_{a} \wedge e^{b}\right) \\
+d\left(\dot{\omega}_{b}{ }_{b} \wedge \star\left(e_{a} \wedge e^{b}\right)\right)= & \left(\dot{\omega}^{a}{ }_{c} \wedge \omega^{c}{ }_{b}-\dot{\omega}^{c}{ }_{b} \wedge \omega^{a}{ }_{c}\right) \wedge \star\left(e_{a} \wedge e^{b}\right) \\
+\dot{\omega}^{a}{ }_{b} \wedge d \star\left(e_{a} \wedge e^{b}\right)+d\left(\dot{\omega}^{a}{ }_{b} \wedge \star\left(e_{a} \wedge e^{b}\right)\right)= & \dot{\omega}^{a}{ }_{b} \wedge \omega^{b}{ }_{c} \wedge \star\left(e_{a} \wedge e^{c}\right) \\
& -\dot{\omega}^{a}{ }_{b} \wedge \omega^{c}{ }_{a} \wedge \star\left(e_{c} \wedge e^{b}\right)+\dot{\omega}_{b}^{a} \wedge d \star\left(e_{a} \wedge e^{b}\right)+d\left(\dot{\omega}_{b}^{a}{ }_{b}\right. \\
= & \dot{\omega}^{a}{ }_{b} \wedge D \star\left(e_{a} \wedge e^{b}\right)+d\left(\dot{\omega}_{b}{ }_{b} \wedge \star\left(e_{a} \wedge e^{b}\right)\right)
\end{aligned}
$$

where $D$ is the exterior covariant derivative and $\omega^{a}{ }_{b}$ denotes the variation of $\omega^{a}{ }_{b}$. Since $\dot{\omega}_{b}{ }_{b}$ has compact support

$$
\int \underbrace{\Lambda_{E H}}_{\omega}=\int \dot{\omega}^{a}{ }_{b} \wedge D \star\left(e_{a} \wedge e^{b}\right)
$$

The coframe variation gives:

$$
\begin{gathered}
\underbrace{\Lambda_{E H}}_{e}=\underbrace{R_{b}^{a} \wedge \star\left(e_{a} \wedge e^{b}\right)}_{e}= \\
R_{b}^{a} \wedge \underbrace{\star\left(e_{a} \wedge e^{b}\right)}_{e}=\delta e^{c} \wedge R_{b}^{a} \star\left(e_{a} \wedge e^{b} \wedge e_{c}\right)
\end{gathered}
$$

because $R_{b}^{a}$ is a coframe-independent object.

We can write:

$$
\underbrace{\Lambda_{E H}}_{e}=\delta e^{c} \wedge G_{c}
$$

where the Einstein $(n-1)$-forms are:

$$
G_{c}=R_{b}^{a} \wedge \star\left(e_{a} \wedge e^{b} \wedge e_{c}\right)
$$


For any coframe-independent $p$-forms $\alpha$ and $\beta$, the following relation holds:

$$
\underbrace{\alpha \wedge \star \beta}_{e}=-\dot{e}^{c} \wedge\left[i_{c} \beta \wedge \star \alpha-(-1)^{p} \alpha \wedge i_{c} \star \beta\right]
$$

This can be proved as follows. Let us write the generic $p$-form $\beta$ as:

$$
\beta=\beta_{a_{1}, a_{2}, \ldots \ldots a_{p}} e^{a_{1} a_{2} \ldots . a_{p}}
$$

¿From the frame-independence of $\beta$ it follows:

$$
\frac{1}{p} \delta_{e}\left(\beta_{a_{1}, a_{2}, \ldots . . a_{p}}\right) e^{a_{1} a_{2} \ldots . . a_{p}}+\beta_{a_{1}, a_{2}, \ldots . . a_{p}}\left(\delta_{e} e^{a_{1}} \wedge e^{a_{2} \ldots a_{p}}\right)=0
$$

Then

$$
\delta_{e}(\star \beta)=\delta_{e}\left(\beta_{a_{1}, a_{2}, \ldots \ldots a_{p}} \star e^{a_{1} a_{2} \ldots . a_{p}}\right)
$$

so that

$$
\begin{aligned}
\underbrace{\alpha \wedge \star \beta}_{e} & =\alpha \wedge \delta\left(\beta_{a_{1}, a_{2}, \ldots \ldots a_{p}} \star e^{a_{1} a_{2} \ldots a_{p}}\right) \\
& =\alpha \wedge \delta_{e}\left(\beta_{a_{1}, a_{2}, \ldots . . a_{p}}\right) \star e^{a_{1} a_{2} \ldots a_{p}}+\alpha \wedge \beta_{a_{1}, a_{2}, \ldots \ldots a_{p}} \delta\left(\star e^{a_{1} a_{2} \ldots . a_{p}}\right) \\
& =\delta_{e}\left(\beta_{a_{1}, a_{2}, \ldots \ldots a_{p}}\right) e^{a_{1} a_{2} \ldots a_{p}} \wedge \star \alpha+(-1)^{p} \delta e^{c} \wedge \alpha \wedge \beta_{a_{1}, a_{2}, \ldots \ldots a_{p}} \star\left(e^{a_{1} a_{2} \ldots a_{p}}{ }_{c}\right) \\
& =-p \beta_{a_{1}, a_{2}, \ldots \ldots a_{p}} \delta e^{a_{1}} \wedge e^{a_{2} \ldots a_{p}} \wedge \star \alpha+(-1)^{p} \delta e^{c} \wedge \alpha \wedge i_{c} \star \beta \\
& =-\delta e^{c} \wedge\left[i_{c} \beta \wedge \star \alpha-(-1)^{p} \alpha \wedge i_{c} \star \beta\right]
\end{aligned}
$$

where use has been made of the property $\alpha \wedge \star \beta=\beta \wedge \star \alpha$ in the third line and of eq. (54) in the fourth line.

¿From eq. (52) we can get a relation between the scalar curvature and the stress forms. To get it, notice that if $\alpha$ and $\beta$ are frame-dependent then we have to add to (52) the coframe variation of $\alpha$ and $\beta$. Let us define $\Delta \tau_{c}$ by:

$$
\delta e^{c} \wedge \Delta \tau_{c}=\underbrace{\alpha}_{e} \wedge \star \beta+\alpha \wedge \star \underbrace{\beta}_{e}
$$

Consider an action of the form:

$$
\Lambda=k R \star 1+\sum_{k} b_{k}\left(\alpha_{k} \wedge \star \beta_{k}\right)
$$


where $\alpha_{k}$ and $\beta_{k}$ are generic $p_{k}$-forms and $b_{k}$ are constants.

The coframe variation of (58) yields the equations:

$$
k R_{b}^{a} \wedge i_{c} i^{b} i_{a} \star 1-\sum_{k} b_{k}\left[i_{c} \beta_{k} \wedge \star \alpha_{k}-(-1)^{p_{k}} \alpha_{k} \wedge i_{c} \star \beta_{k}\right]+\sum_{k} \Delta \tau_{c}[k]=0
$$

where $\Delta \tau_{c}[k]$ is the extra term in the stress forms coming from the generic term $b_{k}\left(\alpha_{k} \wedge \star \beta_{k}\right)$.

By taking the wedge product of (21) with $e^{c}$ we get:

$$
(-1)^{n+1}(n-2) R \star 1+(-1)^{n} \sum_{k}\left[\left(2 p_{k}-n\right) b_{k}\left(\alpha_{k} \wedge \star \beta_{k}\right)+\sum_{k}\left(\Delta \tau_{c}[k]\right) \wedge e^{c}=0\right.
$$

Consider now a situation in which we have an action density of the form:

$$
\Lambda_{E H}+F(\mathbf{e}, \omega)
$$

The connection variation gives the equation:

$$
D \star\left(e^{a} \wedge e_{b}\right)=F_{b}^{a}
$$

with $F^{a}{ }_{b}(n-1)$-forms defined by:

$$
\underbrace{F}_{\omega}=\dot{\omega}_{a}^{b} \wedge F_{b}^{a}
$$

Equation (62) is called the Cartan equation.

¿From (63) it follows

$$
F^{a}{ }_{a}=0
$$

We can define the set of 0 -forms $f^{c a}{ }_{b}$ by

$$
F^{a}{ }_{b}=f^{c a}{ }_{b} \star e_{c}
$$

¿From (64) we get $f^{c a}{ }_{a}=0$.

It is possible to obtain the general solution of the Cartan equation by decomposing both the Weyl form and the torsion into a trace part and a traceless part [32]. We have, respectively:

$$
Q_{a b}=\hat{Q}_{a b}+\frac{1}{n} g_{a b} Q
$$


where $\hat{Q}_{a}^{a}=0$, and analogously

$$
T^{a}=\hat{T}^{a}+\frac{1}{n-1} e^{a} \wedge T
$$

with $T \equiv i_{a} T^{a}$ and $i_{a} \hat{T}^{a}=0$.

Eq. (62) becomes therefore decomposed as:

$$
i_{b} \hat{Q}_{a}{ }^{c}-\delta^{c}{ }_{b} i_{d} \hat{Q}_{a}{ }^{d}+\left(\delta^{c}{ }_{b} \delta^{d}{ }_{a}-\delta^{c}{ }_{a} \delta^{d}{ }_{b}\right)\left(\frac{n-2}{2 n} i_{d} Q-i_{d} i_{h} T^{h}\right)-i_{b} i_{a} T^{c}+f^{c}{ }_{a b}=0
$$

that is,

$$
\begin{aligned}
i_{a} \hat{Q}_{b c}-i_{a} i_{b} T_{c} & =-\frac{1}{2 n} g_{b c} i_{a} Q+\frac{1}{2 n} g_{a c} i_{b} Q-f_{c b a} \\
& -\frac{1}{n(n-2)} g_{a c} f^{d}{ }_{d b}+\frac{n-1}{n(n-2)} g_{b c} f_{d a}^{d} \\
& +\frac{n-1}{n(n-2)} g_{a c} f_{b d}^{d}-\frac{1}{n(n-2)} g_{b c} f_{a d}^{d}
\end{aligned}
$$

Using the symmetry of $\hat{Q}_{a b}$ and the antisymmetry of $i_{a} i_{b} T_{c}$, we find

$$
\begin{gathered}
i_{a} i_{b} \hat{T}_{c}=\frac{1}{n-1}\left(g_{b c} f_{a d}^{d}-g_{a c} f_{b d}^{d}\right)-\frac{1}{2}\left(f_{b a c}+f_{b c a}+f_{c a b}-f_{c b a}-f_{a b c}-f_{a c b}\right) \\
i_{a} \hat{Q}_{b c}=\frac{1}{n} g_{b c}\left(f_{d a}^{d}+f_{a d}^{d}\right)-\frac{1}{2}\left(f_{b a c}+f_{b c a}+f_{c a b}+f_{c b a}-f_{a b c}-f_{a c b}\right)
\end{gathered}
$$

and

$$
T-\frac{n-1}{2 n} Q=\frac{1}{n(n-2)}\left(f_{a c}^{c}+(1-n) f_{c a}^{c}\right) e^{a}
$$

The following expressions hold true for the traceless parts of the torsion and the nonmetricity, respectively:

$$
\begin{gathered}
\hat{T}_{c}=\frac{1}{n-1}\left(e_{c} \wedge e^{a}\right) f_{a d}^{d}-\frac{1}{2}\left(e^{b} \wedge e^{a}\right)\left(f_{b a c}+f_{b c a}+f_{c a b}\right) \\
\hat{Q_{a b}}=\frac{1}{n} g_{a b}\left(f_{d a}^{d}+f_{a d}^{a}\right) e^{a}-\left(f_{b a c}+f_{b c a}-f_{a b c}\right) e^{a}
\end{gathered}
$$

Equations (72)-(74) provide the general solution of the Cartan equation (62). The study of the properties of such a general solution is fundamental in the 
study of properties of non-Riemannian theories of gravitation [33].

Let us notice that in general the connection variation of a generic action gives a Cartan equation which is a differential equation to be solved for the non-Riemannian part of the connection. In the present case, however, the fact that we are considering an action density like (61) allows us to solve algebraically eq. (62) for $\lambda^{a}{ }_{b}$.

\section{Particular Solutions of the Cartan Equa- tion}

In this section we present some particular solutions of the Cartan equation (62). The different cases are classified depending on the explicit expression of the $(n-1)$-forms $F_{b}^{a}$.

In particular, we will prove that, if $F^{a}{ }_{b}$ takes the special form of subsection 1.3.6 or 1.3.8, the traceless part of the torsion $\hat{T}^{a}$ turns out to be zero.

\section{1 $\quad F_{b}^{a}=0$}

The first case we are going to consider is when $F_{b}^{a}=0$.

Then, using the equations of the previous section, we get:

$$
i_{a} \hat{Q}_{b c}=0
$$

that is $\hat{Q}_{b c}=0$ and

$$
i_{a} i_{b} \hat{T}_{c}=0
$$

which means $\hat{T}_{c}=0$. We can write:

$$
\begin{gathered}
T^{a}=\frac{1}{n-1}\left(e^{a} \wedge T\right) \\
T=\frac{n-1}{2 n} Q
\end{gathered}
$$


The non-metricity 1-forms and the torsion 2-forms result to be:

$$
\begin{gathered}
Q_{a b}=\frac{1}{n} g_{a b} Q \\
T^{a}=\frac{1}{2 n}\left(e^{a} \wedge Q\right)
\end{gathered}
$$

The non-Riemannian part of the connection takes the following very simple form:

$$
\lambda_{a b}=-\frac{1}{2 n} Q g_{a b}
$$

so the traceless part of the non-Riemannian part of the connection, defined by

$$
\hat{\lambda}_{b}^{a}=\lambda_{b}^{a}-\frac{1}{n} \lambda_{c}^{c} \delta_{b}^{a}
$$

is zero.

\section{$4.2 f_{c a b}=-f_{c b a}$}

In this case the formulas of sect. 3 yield

$$
\begin{gathered}
\hat{Q}_{a b}=0 \\
T-\frac{n-1}{2 n} Q=\frac{1}{n-2} f^{c}{ }_{a c} e^{a}
\end{gathered}
$$

The traceless part of the torsion 2 -forms is calculated to be

$$
\hat{T}_{c}=\frac{1}{n-1}\left(e_{c} \wedge e^{a}\right) f_{a d}^{d}+\frac{1}{2}\left(e^{b} \wedge e^{a}\right) f_{c b a}
$$

thus providing the solution

$$
\begin{gathered}
Q_{a b}=\frac{1}{n} g_{a b} Q \\
T_{c}=\frac{1}{n-1}\left(e_{c} \wedge e^{a}\right) f_{a d}^{d}+\frac{1}{2}\left(e^{b} \wedge e^{a}\right) f_{c b a}+\frac{1}{n-1} e_{c} \wedge T
\end{gathered}
$$




\section{3 $F_{b}^{a}=\sum_{k}\left(e^{a} \wedge i_{b} \star A_{k}\right)$}

Let us now consider the case in which the forms $F^{a}{ }_{b}$ can be written as:

$$
F_{b}^{a}=\sum_{k}\left(e^{a} \wedge i_{b} \star A_{k}\right)
$$

with $A_{k}$ set of 1 -forms.

One gets easily

$$
f_{c a b}=\left[\sum_{k}\left(i_{c}\left(A_{k}\right) g_{a b}-i_{a}\left(A_{k}\right) g_{c b}\right)\right]
$$

so that

$$
f_{c a b}=-f_{a c b}
$$

Therefore

$$
f_{a c}^{c}=\left[(1-n) i_{a} \sum_{k} A_{k}\right]
$$

and

$$
f^{c}{ }_{c a}=0
$$

The condition $f^{c a}{ }_{a}=0$ implies $(n-1) i^{c} \sum_{k} A_{k}=0$ i.e. $\sum_{k} A_{k}=0$.

Then we have

$$
T=(n-1)\left(\frac{Q}{2 n}-\frac{\sum_{k}\left(A_{k}\right)}{n(n-2)}\right)
$$

The calculation of $Q_{b c}$ gives:

$$
Q_{b c}=\left[\sum_{k}\left(-e_{b} i_{c} A_{k}-e_{c} i_{b} A_{k}+\frac{n+1}{n} g_{b c} A_{k}\right)\right]+\frac{1}{n} g_{b c} Q
$$

Indeed, from the general expression (74), on account of the antisymmetry of $f_{c a b}$, one finds

$$
Q_{b c}=\frac{1}{n} g_{b c} f_{a d}^{d} e^{a}+f_{a b c} e^{a}+f_{a c b} e^{a}+\frac{1}{n} Q g_{b c}
$$

and, from eq. (89):

$$
\begin{aligned}
f_{a d}^{d} e^{a} & =(1-n) \sum_{k} A_{k} \\
f_{a c b} e^{a} & =\left[\sum_{k}\left(A_{k} g_{c b}-i_{c}\left(A_{k}\right) e_{b}\right)\right] \\
f_{a b c} e^{a} & =\left[\sum_{k}\left(A_{k} g_{c b}-i_{b}\left(A_{k}\right) e_{c}\right)\right]
\end{aligned}
$$


Using the previous relations we get the result (94) for $Q_{b c}$. Analogously, from eq. (73) we find

$$
\begin{aligned}
\hat{T}_{c} & =\left[\sum _ { k } \left(\frac{1}{n-1}\left(e_{c} \wedge e^{a}\right)(1-n) i_{a}\left(A_{k}\right)\right.\right. \\
& +\frac{1}{2}\left(e^{b} \wedge e^{a}\right)\left[i_{a} A_{k} g_{b c}-i_{b} A_{k} g_{a c}+i_{c} A_{k} g_{b c}\right. \\
& \left.\left.-i_{b} A_{k} g_{a c}+i_{a} A_{k} g_{b c}-i_{c} A_{k} g_{a b}\right]\right) \\
& \left.=\sum_{k}\left(-\left(e_{c} \wedge A_{k}\right)+e^{b} \wedge\left(e^{a} \wedge i_{a} A_{k}\right) g_{b c}+\left(e^{a} \wedge e^{b}\right) i_{b}\left(A_{k}\right) g_{a c}\right)\right]=\sum_{k}\left(e_{c} \wedge A_{k}\right)
\end{aligned}
$$

so the traceless part of the torsion comes to be:

$$
\hat{T}_{c}=\left[e_{c} \wedge \sum_{k}\left(A_{k}\right)\right]
$$

\section{4 $F_{b}^{a}=\delta^{a}{ }_{b} \star A$}

With $A$ a generic 1 -form in this case:

$$
f_{c a b}=g_{a b} i_{c} A
$$

The condition $f^{c a}{ }_{a}=0$ gives $n i^{c} A=0$.

Notice that:

$$
T-\frac{n-1}{2 n} Q=-\frac{A}{n}
$$

Using the same method of the previous case we get:

$$
\hat{T}_{c}=\frac{n}{n-1}\left(e_{c} \wedge A\right)
$$

so that:

$$
T_{c}=\frac{n+1}{n}\left(e_{c} \wedge A\right)+\frac{1}{2 n}\left(e_{c} \wedge Q\right)
$$

The non-metricity can be calculated using the expression (74) which, on the basis of the symmetry properties of $f_{c a b}$, becomes:

$$
i_{a} \hat{Q}_{b c}=\frac{2}{n} g_{b c} f_{d a}^{d}-f_{b a c}-f_{c a b}+f_{a c b}
$$


or

$$
\hat{Q}_{b c}=\frac{2}{n} g_{b c} f_{d a}^{d} e^{a}-f_{b a c} e^{a}-f_{c a b} e^{a}+f_{a c b} e^{a}
$$

By plugging in the expression for $f_{a b c}$ we get:

$$
\begin{aligned}
\hat{Q}_{b c} & =\left[\frac{2}{n} g_{b c} i_{a}(A) e^{a}-g_{a c}\left(i_{b} A\right) e^{a}\right. \\
& -g_{a b}\left(i_{c} A\right) e^{a}+g_{c b}\left(i_{a} A\right) e^{a} \\
& =\frac{2}{n} g_{b c} A-i_{b} A e_{c}-i_{c} A e_{b}+g_{b c} A \\
& =-e_{c} i_{b} A-e_{b} i_{c} A+\frac{2+n}{n} g_{b c} A
\end{aligned}
$$

and finally

$$
Q_{b c}=\left[-e_{b} i_{c}(A)-e_{c} i_{b}(A)+\frac{n+2}{n} g_{b c} A\right]+\frac{1}{n} Q g_{b c}
$$

The last two cases are useful to understand the relation between certain models of non-Riemannian gravity and Einstein theory [33].

\section{5 $\quad F_{b}^{a}=e^{a} \wedge \star A_{b}, i^{b} A_{b}=0$}

Here $A_{b}$ is a 2 -form, wich can be assumed to be traceless without loss of generality. Indeed, a not traceless $A_{b}$ can be written as:

$$
A_{b}=\hat{A}_{b}+\frac{1}{n-1}\left(e_{b} \wedge A\right)
$$

with $A=i^{a} A_{a}$. The contribution from the second term gives a term of the type already treated in 4.3 , so we can limit ourselves to considering the traceless case.

It is easy to find that:

$$
f_{c a b}=i_{a} i_{c} A_{b}
$$

whence

$$
\begin{aligned}
f_{c a b} & =-f_{a c b} \\
f^{c a}{ }_{a} & =i^{c} i^{a}\left(A_{a}\right)=0 \\
f^{c c}{ }_{a} & =0 \\
f^{c a}{ }_{c} & =i^{a} i^{c}\left(A_{c}\right)=0
\end{aligned}
$$


We get therefore

$$
T-\frac{n-1}{2 n} Q=0
$$

In this case the relation between $Q$ and $T$ is the same as (78).

After some calculations we find:

$$
\hat{Q}_{a b}=-\left[i_{a}\left(A_{b}\right)+i_{b}\left(A_{a}\right)\right]
$$

and

$$
\hat{T}_{c}=-\left[e^{a} \wedge i_{c}\left(A_{a}\right)+A_{c}\right]
$$

which means

$$
e^{c} \wedge \hat{T}_{c}=e^{a} \wedge A_{a}
$$

If we consider the case in which $A_{b}$ can be written as

$$
A_{b}=i_{b} B
$$

with $B$ a 3-form, then $\hat{Q}_{a b}=0$.

Another interesting case is when $A_{b}$ coincides with $\hat{T}_{b}$ apart from a constant factor $\lambda$ : $A_{b}=\lambda \hat{T}_{b}$. Then, it follows from relation (113) that, if $\lambda=1, \hat{T}_{c}$ is arbitrary, while if $\lambda \neq 1$ we need:

$$
e^{c} \wedge \hat{T}_{c}=0
$$

\section{6 (case $3.3+3.4) F^{a}{ }_{b}=\delta^{a}{ }_{b} \star A+\sum_{k}\left(e^{a} \wedge i_{b} \star A_{k}\right)$}

We get:

$$
f_{c a b}=\sum_{k}\left(\left(i_{c} A_{k}\right) g_{a b}-\left(i_{a} A_{k}\right) g_{c b}\right)+g_{a b}\left(i_{c} A\right)
$$

The condition $f^{c a}{ }_{a}=0$ gives:

$$
(n-1) \sum_{k} A_{k}+n A=0
$$

Therefore

$$
T=\frac{n-1}{2 n} Q-\left[\frac{n-1}{n(n-2)} \sum_{k} A_{k}+\frac{1}{n} A\right]
$$


The non-metricity reads:

$Q_{b c}=\left[-e_{b} i_{c}\left(\sum_{k} A_{k}+A\right)-e_{c} i_{b}\left(\sum_{k} A_{k}+A\right)+\frac{n+1}{n} g_{b c} \sum_{k} A_{k}+\frac{n+2}{n} g_{b c} A\right]+\frac{1}{n} Q g_{b c}$

or, by using relation (118):

$$
Q_{b c}=\left[-e_{b} i_{c}\left(\sum_{k} A_{k}+A\right)-e_{c} i_{b}\left(\sum_{k} A_{k}+A\right)+\frac{2}{n}\left(\sum_{k} A_{k}+A\right) g_{b c}\right]+\frac{1}{n} g_{b c} Q
$$

By putting $A_{1}=-\left(\sum_{k} A_{k}+A\right)$ we can write:

$$
Q_{b c}=e_{b} i_{c} A_{1}+e_{c} i_{b} A_{1}-\frac{2}{n} A_{1} g_{b c}+\frac{1}{n} g_{b c} Q
$$

The traceless part of the torsion is found from (73) and (118):

$$
\hat{T}_{c}=\left[e_{c} \wedge \sum_{k} A_{k}+\frac{n}{n-1} e_{c} \wedge A\right]=0
$$

whence

$$
T^{c}=\frac{1}{n-1}\left(e^{c} \wedge T\right)
$$

$4.7 \quad F_{b}^{a}=\sum_{k}\left(e_{b} \wedge i^{a} \star A_{k}\right)$

In this case we get:

$$
f_{c a b}=\left[\sum_{k}\left(i_{c}\left(A_{k}\right) g_{a b}\right)-i_{b}\left(A_{k}\right) g_{a c}\right]
$$

and therefore

$$
f_{c a b}=-f_{b a c}
$$

We see that $f^{c}{ }_{a c}=0$ and:

$$
f_{c a}^{c}=\left[(1-n) i_{a} \sum_{k} A_{k}\right]
$$


The condition $f^{c a}{ }_{a}=0$ implies $(n-1) i^{c} \sum_{k} A_{k}=0$, so that

$$
T-\frac{n-1}{2 n} Q=\sum_{k}\left[\frac{(n-1)^{2}}{n(n-2)} A_{k}\right]
$$

The calculation of $Q_{b c}$ yields the same expression already found for the case 4.3:

$$
Q_{b c}=\left[\sum_{k}\left(-e_{b} i_{c} A_{k}-e_{c} i_{b} A_{k}+\frac{n+1}{n} g_{b c} A_{k}\right)\right]+\frac{1}{n} g_{b c} Q
$$

As far as the traceless part of the torsion is concerned, one finds

$$
\hat{T}_{c}=\frac{1}{2}\left(e^{b} \wedge e^{a}\right) f_{b c a}=-\left(e^{b} \wedge e^{a}\right) g_{c a} \sum_{k} i_{b} A_{k}=e^{c} \wedge \sum_{k} A_{k}
$$

i.e. the same expression of the case 4.3.

\section{$4.8($ case $3.7+3.4) F_{b}^{a}=\delta_{b}^{a} \star A+\sum_{k}\left(e_{b} \wedge i^{a} \star A_{k}\right)$}

We have:

$$
f_{c a b}=\left[\sum_{k}\left(i_{c}\left(A_{k}\right) g_{a b}-i_{b}\left(A_{k}\right) g_{a c}\right)+g_{a b} i_{c} A\right.
$$

It is easy to see that all the results of the case 4.6 still hold, apart from the relation between $T$ and $Q$ which is modified into:

$$
T=\frac{n-1}{2 n} Q+\left[\frac{(n-1)^{2}}{n(n-2)} A_{k}-\frac{A}{n}\right]
$$

The expression for $Q_{b c}$ and $T_{c}$ are the same, whereas the traceless part of the torsion 2-forms comes out to be zero.

\section{Applications: Proca theories from Metric Affine gravity}

In this section we want to show briefly how, by using the findings of the previous section, a quite general model in non-Riemannian gravity gives a 
Proca-type equation for the Weyl form $Q$. Such a result is fundamental in proving the Obukhov theorem [34,35], and therefore the content of this section is more than a simple application of the formalism.

Let us consider a model in which the action is:

$$
\begin{aligned}
S= & \int k R \star 1+\frac{c_{1}}{2}(d Q \wedge \star d Q)+\frac{c_{2}}{2}(Q \wedge \star Q)+\frac{c_{3}}{2}(Q \wedge \star T) \\
& +\frac{c_{4}}{2}(T \wedge \star T)+\frac{c_{5}}{2}\left(T^{c} \wedge \star T_{c}\right)+\frac{c_{6}}{2}(\mathcal{Q} \wedge \star Q)+ \\
& \frac{c_{7}}{2}(\mathcal{Q} \wedge \star T)+\frac{c_{8}}{2}(\mathcal{Q} \wedge \star \mathcal{Q})
\end{aligned}
$$

where:

$$
\mathcal{Q}=e^{a} i^{b} Q_{a b}
$$

We want to show that, irrespective of the values of coefficients $c_{1}, \ldots c_{8}$, one gets a Proca equation for $Q$.

Let us derive the connection variation of all terms in the action (132). The results one easily gets $(\bmod d)$ are as follows:

Terms $d Q \wedge \star d Q,(Q \wedge \star Q),(T \wedge \star T),\left(T^{c} \wedge \star T_{c}\right):$

$$
\delta \omega^{a}{ }_{b} \wedge\left[2 \delta^{b}{ }_{a}\left(c_{1} d \star d Q+c_{2} \star Q\right)+c_{4}\left(e^{b} \wedge i_{a} \star T\right)-c_{5}\left(e^{b} \wedge \star T_{a}\right)\right]
$$

$\operatorname{Term} \frac{c_{3}}{2}(Q \wedge \star T):$

$$
-2 \delta \omega_{b}^{a} \delta_{b}^{a} \wedge \star T-\delta \omega^{a}{ }_{b} \wedge\left(e^{b} \wedge i_{a} \star Q\right)
$$

$\operatorname{Term} \mathcal{Q} \wedge \star \mathcal{T}:$

$$
\begin{aligned}
& -2 \delta \omega^{a}{ }_{b} \delta^{a}{ }_{b} \wedge \star T-\delta \omega^{a}{ }_{b} \wedge\left(e^{b} \wedge i_{a} \star \mathcal{Q}\right)+ \\
& \delta \omega^{a}{ }_{b} \wedge\left(e_{a} \wedge i^{b} \star T\right)+\delta \omega^{a}{ }_{b} \wedge\left(e^{b} \wedge i_{a} \star T\right)
\end{aligned}
$$

$\operatorname{Term} \mathcal{Q} \wedge \star Q:$

$$
-2 \delta \omega^{a}{ }_{b} \delta^{b}{ }_{a} \wedge \star(Q+\mathcal{Q})+\delta \omega^{a}{ }_{b} \wedge\left(e_{a} \wedge i^{b} \star Q\right)+\delta \omega^{a}{ }_{b} \wedge\left(e^{b} \wedge i_{a} \star Q\right)
$$




\section{$\operatorname{Term} \mathcal{Q} \wedge \star \mathcal{Q}:$}

$$
-4 \delta \omega^{a}{ }_{b} \delta^{b}{ }_{a} \wedge \star \mathcal{Q}+2 \delta \omega^{a}{ }_{b} \wedge\left(e_{a} \wedge i^{b} \star \mathcal{Q}\right)+2 \delta \omega^{a}{ }_{b} \wedge\left(e^{b} \wedge i_{a} \star \mathcal{Q}\right)
$$

The equation we get from the connection variation will split in a trace part and a traceless part.

All the terms considered in (134)-(138) are of the type considered in section 4. Then, it is easy to check that in general the trace part will contain terms like

$$
\lambda_{1} \star T+\lambda_{2} \star Q+\lambda_{3} \star \mathcal{Q}
$$

where $\lambda_{1}, \lambda_{2}, \lambda_{3}$ are constants.

Then we can write:

$$
c_{1} d \star d Q+c_{2} \star Q=\lambda_{1} \star T+\lambda_{2} \star Q+\lambda_{3} \star \mathcal{Q}
$$

where $\lambda_{1}, \lambda_{2}, \lambda_{3}$ are constants related to $c_{3}, c_{4}, \ldots c_{8}$.

We can get other two independent relations from the condition $f^{c a}{ }_{a}=0$ and from (72):

$$
T-\frac{n-1}{2 n} Q=\frac{1}{n(n-2)}\left(f_{a c}^{c}+(1-n) f_{c a}^{c}\right) e^{a}
$$

Exploiting the relations of the previous sections it is possible to verify that these two conditions in general will give two independent linear relations between $Q, T, \mathcal{Q}$ which can be used to eliminate $T, \mathcal{Q}$ in eq. (140).

The conclusion is that Eq. (140) can be written as:

$$
c_{1} d \star d Q+\left(c_{2}+\Delta c_{2}\right) \star Q=0
$$

where $\Delta c_{2}$ will in general depend on the constants $c_{3}, c_{4}, \ldots . . c_{8}$.

Therefore, the Proca-type behaviour for $Q$ is proved. Also the fields $T, \mathcal{Q}$ will satisfy Proca-type equations.

Let us observe that by choosing the constants $c_{3}, c_{4}, \ldots . c_{8}$ in a proper way we can satisfy the condition:

$$
c_{2}+\Delta c_{2}=0
$$

so that we get a Maxwell-like equation for $Q$ :

$$
d \star d Q=0
$$


Such a result can be used to get exact solutions in Metric Affine Gravity from known solutions of Einstein-Maxwell theory [36,37].

\section{ACKNOWLEDGMENTS}

One of us (R.S.) thanks the NOOPOLIS Foundation, Italy, for financial sup-

port, and R. Tucker and C. Wang for stimulating discussions on the topic. 


\section{REFERENCES}

1] E. Kroener, Continuum theory of defects, in Physics of Defects, Les Houches Session XXXV, 1980, R. Balian et al. eds. (North-Holland, Amsterdam 1981), p. 215.

2] E. Kroener, The role of differential geometry in the mechanics of solids, in Proc. 5th Nat. Congr. Theor. Appl. Mech., Vol. 1, (Bulgarian Acad. Sci. Sofia 1985), p. 352 .

3] Y. Neeman, D. Sijacki, Ann. Phys. 120 (1979) 292.

4] Y. Neeman, D. Sijacki, Phys. Rev. D 37 (1988) 3267.

5] P. J. Steinhart, Class. Quant. Grav. 10 (1993) S33.

6] J. Scherk and J. H. Schwartz, Phys. Lett. B 52B (1974) 347.

7] T. Dereli, R. W. Tucker, Class. Quant. Grav. 12 L31 (1995).

8] T. Dereli, M. Onder, R. W. Tucker, Class. Quant. Grav. 12 L25 (1995).

9] T. Dereli, R. Tucker, Class. Quant. Grav. 11 (1994) 2575.

10] F. W. Hehl, E. Lord, L. L. Smalley, Gen. Rel. Grav. 13 (1981) 1037.

11] F. W. Hehl, E. W. Mielke: Non-metricity and Torsion, Proc. 4th Marcel Grossman Meeting on General relativity, Part A, ed. R. Ruffini ( North Holland, Amsterdam, 1986) p. 277.

12] V. N. Ponomariev, Y. Obukhov, Gen. Rel. Grav. 14 (1982) 309.

13] A. A. Coley, Phys. Rev. D, 27 (1983) 728.

14] A. A. Coley, Phys. Rev. D. 28 (1983) 1829. 
15] A. A. Coley, Nuovo Cim. B69 (1982) 89.

16] M. Gasperini, Class. Quant. Grav. 5 (1988) 521.

17] J. Stelmach, Class. Quant. Grav. 8 (1991) 897.

18] A. K. Aringazin, A. L. Mikhailov, Class. Quant. Grav. 8 (1991) 1685.

19] J. P. Berthias. B. Shabid-Saless, Class. Quant. Grav. 10 (1993) 1039.

20] L. L. Smalley, Phys. Rev. D 21 (1980) 328.

21] R. Tucker, C. Wang, Class. Quant. Grav. 15 (1998) 933.

22] F. W. Hehl, J. D. McCrea, E. W. Mielke, Y. Neeman. Phys. Rep. 2581 (1995).

23] Yu. Obukhov, V. N. Ponomariev, V.V. Zhytnikov, Gen. Rel. Grav. 21 (1989) 1107.

24] J. F. Pascual-Sanchez, Phys. Lett. A 108 (1985) 387.

25] D. W. Sciama, Rev. Mod. Phys. 36 (1964) 463 and 1103.

26] H. Weyl, Geometrie und Physik, Naturwissenschaften, 19 (1931) 49.

27] R. Utiyama, Phys. Rev. 101 (1956) 1597.

28] T. W. B. Kibble, J. Math. Phys. 2 (1961) 212.

29] C. Wang, Ph. D. thesis (Lancaster) (1996).

30] R. Tucker, C. Wang, Non Riem. Grav. Interac., Mathematics of Gravitation, Banach Centre Publications, Vol. 41 (Warsawa ,1997).

31] R. W. Tucker, C. Wang, Class. Quant. Grav. 12 (1995) 2587. 
32] J. D. McCrea, Class. Quant. Grav. 9 (1992) 553.

33] R. Scipioni, Ph. D. Thesis (Lancaster) (1999).

34] Yu. N. Obukhov, E. J. Vlachynsky, W. Esser and F. W. Hehl, Phys. Rev. D 56 (1997) 7769.

35] R. Scipioni, J. Math. Phys. 415 (2000).

36] T. Dereli, M. Onder. J. Schray, R. W. Tucker, C. Wang, Class. Quant. Grav. 13 (1996) L103.

37] F. W. Hehl, A. Macias, Int. J. Mod. Phys. D, 84 (1999) 399. 